\title{
SRSF1 promotes the inclusion of exon 3 of SRA1 and the invasion of hepatocellular carcinoma cells by interacting with exon 3 of SRA1pre-mRNA
}

Sijia Lei ${ }^{1,2}$, Bin Zhang ${ }^{1,2}$, Luyuan Huang ${ }^{3}$, Ziyou Zheng ${ }^{1,2}$, Shaohan Xie ${ }^{1,2}$, Lianghua Shen ${ }^{1,2}$, Mason Breitzig ${ }^{4,5}$, Alexander Czachor ${ }^{4}$, Hongtao Liu ${ }^{6}$, Huiru Luo ${ }^{1,2}$, Yanxia Chen ${ }^{1,2}$, Kangshou Liu ${ }^{7}$, Hanxiao Sun ${ }^{1}$, Qing Zheng ${ }^{1}$, Qiang $\mathrm{Li}^{7}$ and Feng Wang ${ }^{1,2,4}$

\begin{abstract}
Steroid receptor RNA activator 1 (SRA1) has been described as a novel transcriptional co-activator that affects the migration of cancer cells. Through RT-PCR, we identified that skipping exon 3 of SRA1 produces two isoforms, including the truncated short isoform, SRA1-S, and the long isoform, SRA1-L. However, the effect of these two isomers on the migration of HCC cells, as well as the specific mechanism of exon 3 skipping remain unclear. In this study, we found up regulated expression of SRSF1 and SRA1-L in highly metastatic HCCLM3, as well as in HCCS with SRSF1 demonstrating the strongest correlation with SRA1-L. In contrast, we observed a constitutively low expression of SRA1$\mathrm{S}$ and SRSF1 in lowly metastatic HepG2 cells. Overexpression of SRSF1 or SRA1-L promoted migration and invasion by increasing the expression of CD44, while SRA1-S reversed the effect of SRSF1 and SRA1-L in vitro. In addition, lung metastasis in mice revealed that, knockdown of SRSF1 or SRA1-L inhibited the migration of HCC cells, while SRA1-L overexpression abolished the effect of SRSF1 knockout and instead promoted HCC cells migration in vivo. More importantly, RNA immunoprecipitation and Cross-link immunoprecipitation analyses showed that SRSF1 interacts with exon 3 of SRA1 to up regulate the expression of SRA1-L in HCC cells. RNA pull-down results indicated that SRSF1 could also bind to exon 3 of SRA1 in vitro. Finally, minigene -MS2 mutation experiments showed that mutation of the SRA1 exon 3 binding site for SRSF1 prevented the binding of SRA1 pre-mRNA. In summary, our results provide experimental evidence that SRA1 exon 3 inclusion is up regulated by SRSF1 to promote tumor invasion and metastasis in hepatocellular carcinoma.
\end{abstract}

\section{Introduction}

Liver cancer is a common cancer in the world. Many deaths of cancer patients are caused by liver cancer ${ }^{1}$. In $90 \%$ of solid cancer cases, metastasis is the main reason for

Correspondence: Qiang Li (liqiang@jnu.edu.cn) or

Feng Wang (jnubioPharm@126.com)

${ }^{1}$ Institute of Genomic Medicine, College of Pharmacy, Jinan University, Guangzhou, China

${ }^{2}$ International Cooperative Laboratory of Traditional Chinese Medicine Modernization and Innovative Drug Development of Chinese Ministry of Education (MOE), College of Pharmacy, Jinan University, Guangzhou, China Full list of author information is available at the end of the article These authors contributed equally: Sijia Lei, Bin Zhang, Luyuan Huang Edited by Ivano Amelio mortality ${ }^{2}$. It can be seen that inhibiting tumor metastasis is an effective way to improve the survival rate of patients. More specifically, studies have shown that alternative splicing (AS) plays an important role in cancer metastasis ${ }^{3-6}$. Although many abnormal alternative splicing events of mRNA have been reported, few articles have reported on alternative splicing events that simultaneously producecoding proteins isoforms and non-coding isoforms ${ }^{7}$. Therefore, further study of alternative splicing will improve our understanding of liver cancer metastasis and promote the development of more effective targeted therapies.

SRA1 was first described as a novel transcriptional coactivator in 1999 (Accession number: AF092038) and

\section{(c) The Author(s) 2021}

(c) (i) Open Access This article is licensed under a Creative Commons Attribution 4.0 International License, which permits use, sharing, adaptation, distribution and reproduction cc) in any medium or format, as long as you give appropriate credit to the original author(s) and the source, provide a link to the Creative Commons license, and indicate if changes were made. The images or other third party material in this article are included in the article's Creative Commons license, unless indicated otherwise in a credit line to the material. If material is not included in the article's Creative Commons license and your intended use is not permitted by statutory regulation or exceeds the permitted use, you will need to obtain permission directly from the copyright holder. To view a copy of this license, visit http://creativecommons.org/licenses/by/4.0/. 
originally characterized as a non-coding $\mathrm{RNA}^{8}$ responsible for transcriptional co-activation of steroid hormone receptors ${ }^{9}$. Further investigations identified additional SRA1 ncRNA isoforms produced by alternative splicing or multiple transcription start sites ${ }^{10}$. Currently, there are three main isoforms of SRA1 ncRNA: (1) retention of intron 1, (2) alternative $3^{\prime}$ splicing site of exon 2, and (3) deletion of exon $3^{10-12}$. Interestingly, in HCC cells, we only detected the isoform with the deletion of exon 3 in LO2, HepG2, and HCCLM3 cells.

Previous studies suggested that SRA-mediated co-activation is executed by distinct RNA motifs. These studies identified five substructures, which were important for SRA1 co-activation ${ }^{9}$. The deletion of exon 3 would disrupt the formation of two RNA motifs: STR-9 and STR-10. $S R A 1-S$, with a deletion of exon 3 of SRA1-L, mediated the ability of co-activation probably unlike SRA1-L. Therefore, whether SRA1-L and SRA1-S have different functions due to the deletion of exon 3 needs to be further explored. Also, SRA1 stimulates proliferation and apoptosis in vivo ${ }^{13}$. At present, it is not clear whether there are functional differences between SRA1-L and SRA1-S in the proliferation and metastasis of hepatocellular carcinoma cells.

The SR splicing factor family consists of 12 members. Each member contains one or two RNA recognition motifs (RRM) and an RS domain. SR protein can participate in splicing, and can also participate in RNA transcription, export, translation and degradation ${ }^{14}$. SRSF1 is a member of SR splicing factor family. The expression of SRSF1 is related to the occurrence, development and treatment response of tumor, and SRSF1 is highly expressed in tumor cells ${ }^{15}$. SRSF1 can affect tumorigenesis by regulating the alternative splicing of target genes. For examble, For example, SRSF1 regulates PTPMT1 alternative splicing to affect the development of lung cancer ${ }^{16}$. SRSF1 can also regulate BIN1 alternative splicing to affect tumorigenesis ${ }^{17}$. In this study, we found that SRSF1 can regulate the alternative splicing of SRA1 in hepatocellular carcinoma cells, but the specific mechanism is not clear.

Oxisome proliferator-activated receptor (peroxisome proliferators-activated receptor, PPAR) is a kind of nuclear transcription factors activated by ligands, which belongs to the nuclear receptor superfamily. Many articles have reported that PPAR- $\gamma$ is related to the occurrence and development of tumors ${ }^{18-20}$. It is reported that SRA1 can combine with PPAR- $\gamma^{21}$. At present, it has not been found whether there is a difference in the binding ability of SRA1-L and SRA1-S with PPAR- $\gamma$.

Here, we used cell migration experiment and nude mouse metastasis model to determine the role of SRSF1 and SRA1 in HCC cells metastasis. At the same time, we verified the potential binding sites of SRSF1 and SRA1 pre-mRNA through RNA pull-down and other experiments. In addition, through immunoprecipitation, we found that SRA1 isoforms (SRA1-L, SRA1-S) have different binding capabilities with PPAR- $\gamma$. Finally, our results confirmed that SRSF1 is a protein associated with metastasis, and SRA1 is the splicing target of SRSF1, which regulates the incorporation of exon 3 to promote lung cancer cell metastasis. Understanding the mechanism underlying the alternative splicing of SRA1 can provide novel insight into the identification of new therapeutic targets.

\section{Results}

SRA1-L expression was increased in higher-metastatic HCC cells

Firstly, we selected several genes related to tumor metastasis through the NCBI database and literature, including SRA1, DBF4 zinc finger B $(D B F 4 B)$, ZNFX1 antisense RNA 1 (ZFAS1), colorectal neoplasia differentially expressed (CRNDE), endoplasmic reticulum lectin (OS9), and others. Then, we detected the alternative splicing of these genes in HepG2 and HCCLM3 cells by RT-PCR. The results showed that compared with HepG2 cells, in HCCLM3 cells, the exon inclusion rate of DBF4B did not change significantly. Only the long isomer of hnRNPDL, SNHG6 and the short isomer of SPAG9 were detected in HCC cells. More importantly, the SRA1 exon 3 inclusion rate of HCCLM3 cells was significantly higher than that of HepG2 cells (Fig. 1A). The results of RT-PCR and qPCR also showed that in high-metastatic HCC cells, including HCCLM3 and MHCC-97H, the proportion of $S R A 1-L$ isoforms was higher than that of low-metastasis HepG2 HCC cells and normal liver cells (LO2) (Fig. 1B, C). From the detection of clinical samples of HCCs, we also found that the expression of SRA1-L in HCCs was higher than that in adjacent tissues (Fig. 1D).

\section{In contrast to SRA1-S, SRA1-L promotes the migration of HCC cells}

SRA1 has been reported to regulate cell invasion and migration $^{22,23}$. We posited that the two SRA1 isoforms might have different effects. To test this, we first analyzed SRA1 expression in 371 liver hepatocellular carcinoma (LIHC) tissues and 50 normal tissues from UALCAN database $^{24}$. Expression of SRA1 was found to significantly increase in liver cancer (Fig. 2A). Subsequently, we overexpressed SRA1-L and SRA1-S, and designed an independent shRNA targeted against exon 3 for isoform-specific knockdown of SRA1-L; shRNA targeted against exon 2/exon 4 (E2E4) splice junction for isoform-specific knockdown of SRA1-S and an shRNA to selectively target the Intron 1 in HepG2 cells (Fig. 2B). The overexpression and knockdown efficiency of SRA1 isomers have been verified (Fig. S5A, B). In cell proliferation, the results showed that knockdown of SRA1-L could significantly inhibit the proliferation of 


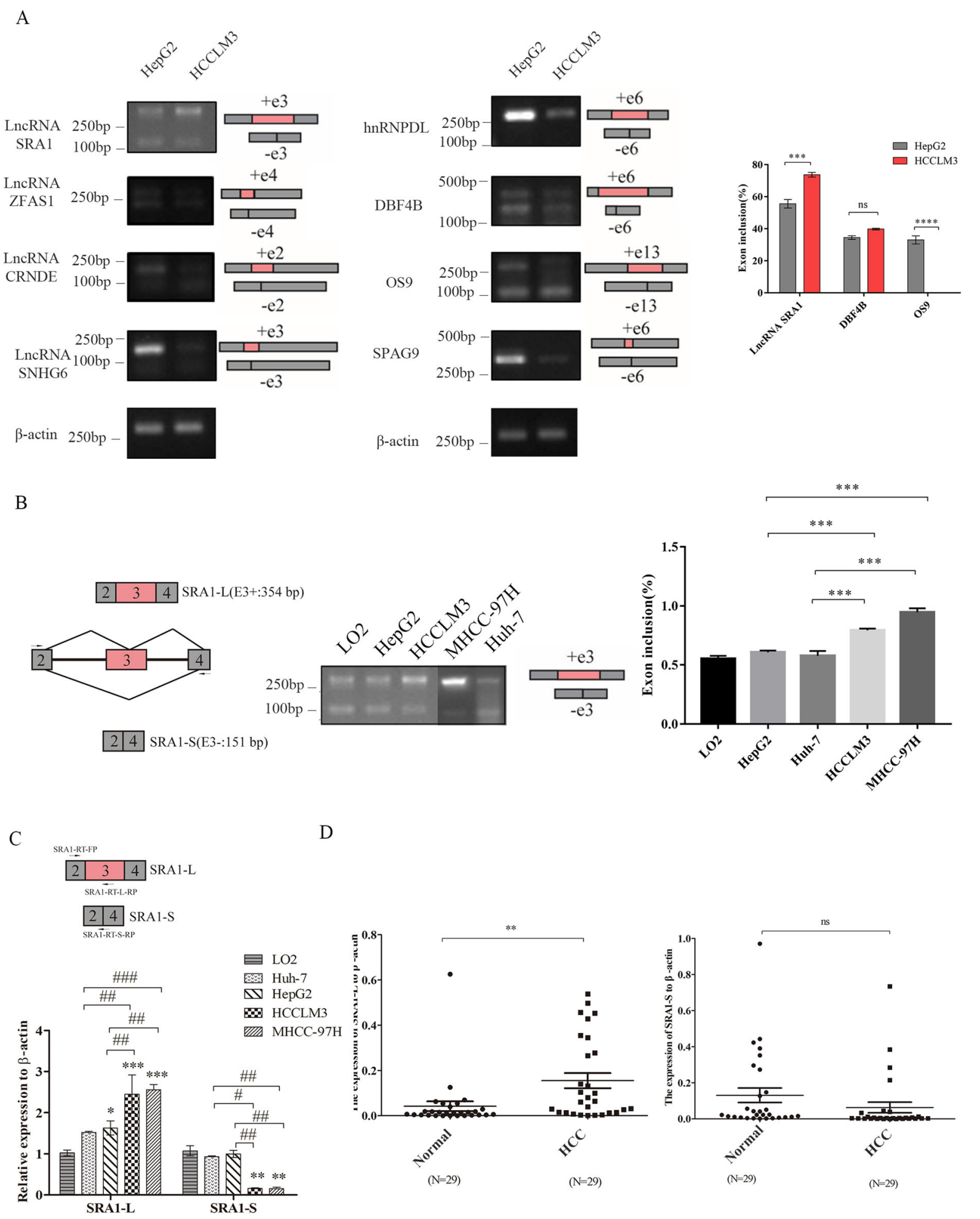

Fig. 1 Differential expression of SRA1 isoforms in Hepatocellular carcinoma cells. A Alternative splicing of the different genes in HepG2 and HCCLM3 by RT-PCR. B The expression of SRA1-L was examined in LO2, Huh-7, HepG2 and HCCLM3, MHCC-97H by RT-PCR. C The expression of SRA1-L was examined in LO2, Huh-7, HepG2 and HCCLM3, MHCC-97H by RT-qPCR. D RT-qPCR was used to detect the expression of SRA1-L and SRA1-S in HCCS. Data from the experiments with one experimental group were presented as means \pm SD (all experiments were performed in triplicate or more). Data are presented as mean \pm S.D. $(N=3)$. The ${ }^{\prime *},{ }^{* *},{ }^{* * *}$ indicate " $P<0.05,0.01,0.001^{\prime \prime}$ versus the control group, respectively. 
A

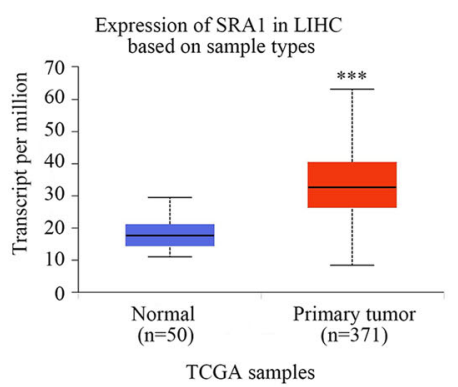

C

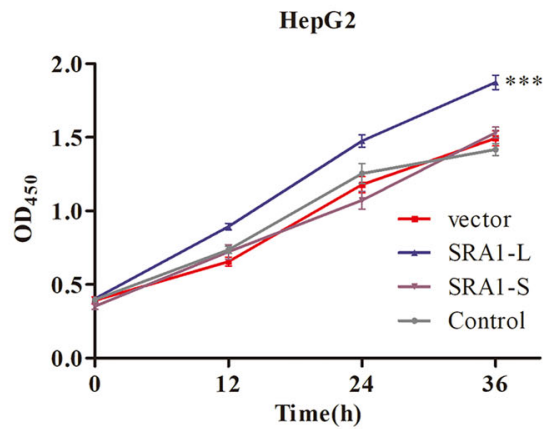

HepG2

D

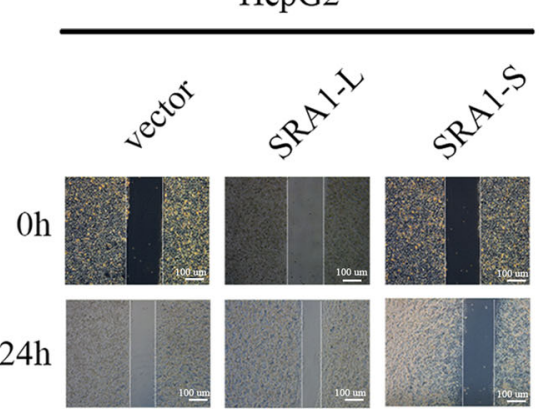

F

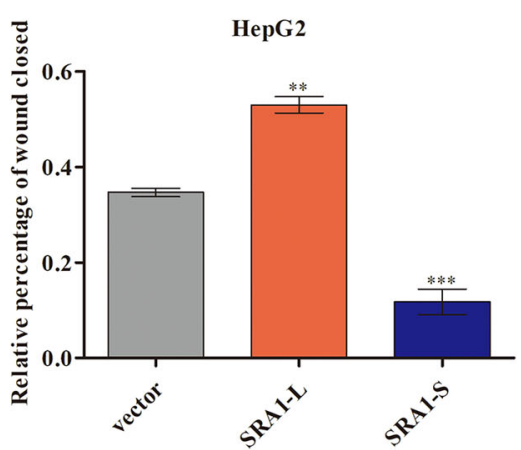

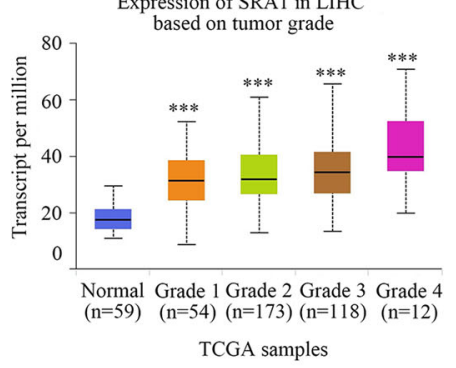

sh-SRA1-E3 position \begin{tabular}{l|l|l|l} 
Exon2 & Exon3 & Exon4 \\
\hline
\end{tabular}

sh-SRA1-E2E4 position

\begin{tabular}{|l|l|}
\hline Exon2 & Exon4 \\
\hline
\end{tabular}

sh-SRA1-In1 position

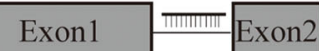

HepG 2
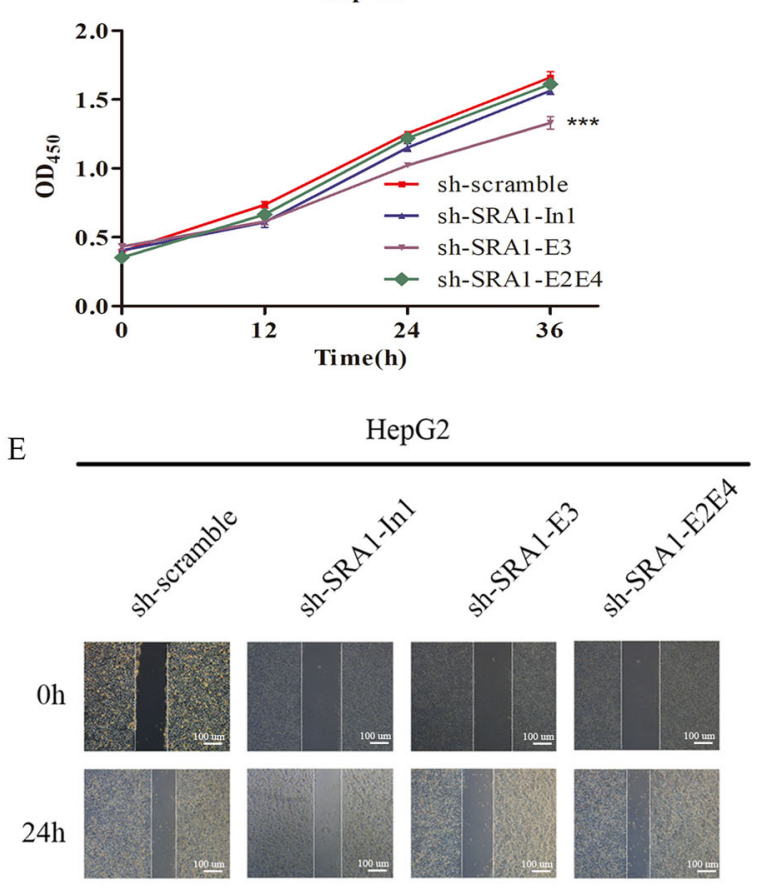

G

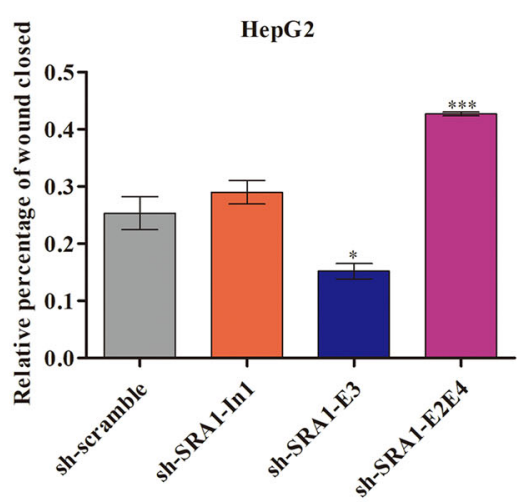

Fig. 2 The isoforms of SRA1 have different effects. A SRA1 expression levels in human HCC tissues from TCGA samples. B Design of shRNA applied to selectively target the In1, exon 3 and exon 2/exon 3 splice junctions. C CCK8 assays were carried out to examine the effect on cell proliferation of SRA1-L/S in HepG2. D, F Wound healing experiments were carried out to estimate the effects of overexpressed SRA1-L/S on the migration in HepG2. E, G Wound healing experiments were carried out to estimate the effects of knocked-down SRA1-E3, SRA1-E2E4, and SRA1-In1 on the migration in HepG2. Data are presented as mean \pm S.D. $(N=3)$. The "****, ***" indicate "P $<0.05,0.01,0.001$ " versus the control group, respectively. "\#, \#\#, \#\#\#" represents " $P<0.05,0.01,0.001 "$. 
HepG2 cells (Fig. 2C). Increasing the expression of SRA1-L can significantly promote the proliferation of HepG2 cells. Also, overexpression or the depletion of SRA1-S has no significant effects on cell proliferation of HepG2 cells (Fig. 2C). The results of wound healing showed overexpressed $S R A 1-L$ promoted the migratory abilities of HepG2 cells, while overexpressed SRA1-S inhibited the migratory abilities of HepG2 cell (Fig. 2D-F). Consistent with the above results, knockdown SRA1-L inhibits the migratory abilities of HepG2 cell, meanwhile, knockdown SRA1-S promotes the migratory abilities of HepG2. (Fig. 2E, G). In cell invasion, knockdown of SRA1-L significantly decreased the invasive ability of hepatoma cells while knockdown of SRA1-In1 had no significant impact. Meanwhile, knockdown of SRA1-S increased the invasive ability of hepatoma cells (Fig. 5E, G). Over-expressing SRA1-L increased the invasive ability of hepatoma cells, but overexpressing SRA1-S decreased the invasive ability of hepatoma cells (Fig. 5F, H).

\section{SRA1-L promotes the migration of HCC cells by increasing} the level of CD44 transcription

SRA1 was a novel transcriptional co-activator, which can regulate the transcription of target genes. Our result of Luciferase Reporter Assays shows that SRA1-L can promote the transcription of $C D 44$, but $S R A 1-S$ has no significant effect on the transcription of CD44 (Fig. 3A). According to the literature, CD44 can promote tumor metastasis through the regulation of ERK and AKT pathway $^{25,26}$, and our results indicate that over-expression of SRA1-L promotes the expression of $\mathrm{p}-\mathrm{AKT}, \mathrm{CD} 44$ and p-ERK, knockdown SRA1-L decreased the expression of p-AKT, CD44 and p-ERK. But SRA1-S does not affect on the expression of p-AKT, CD44 and p-ERK (Fig. 3E-H).

In addition, Luciferase Reporter Assays shows that SRA1- $L$ can inhibit the transcription of $C D H 1$, knockdown $S R A 1-L$ decreased the transcription of $C D H 1$ (Fig. 3B).

\section{SRSF1 is the major regulator for SRA1 exon 3 splicing}

SR proteins and hnRNP proteins are well-known splicing factors that have major roles in the regulation of AS. To find the potential splicing factors regulating the alternative splicing of exon 3 of SRA1, firstly, using public datasets from UALCAN ${ }^{24}$, we examined the connection between the expression of splicing factors and the major cancer stages of liver cancer. Compared with the normal group, the expression of SRSF1, SRSF11 was found to significantly increase in the primary tumor of liver cancer (Fig. S1A). Then, we examined the expression of the SRSF family, the hnRNP family, and other splicing factors in LO2, HepG2, and HCCLM3 cells by RT-qPCR. The results showed that the expression of SRSF1, and SRSF11 in HCCLM3 was significantly higher than that of HepG2 and LO2 (Fig. 4A). Also, in 29 clinical samples of HCCs, we detected the mRNA expression of SRSF1, SRSF8, and SRSF11. The results showed that the expression of SRSF1 in HCCs was higher than that in normal tissue (Fig. 4B). Then, in order to investigate whether SRSF1, and SRSF11 have an effect on the alternative splicing of SRA1. We analyzed the correlation between the expression levels of SRSF1, and SRSF11 with SRA1-L in 29 pairs of HCCs. The results show that the expression levels of SRSF1, and SRSF11 are positively correlated with SRA1-L. The correlation coefficient of SRSF1 is $0.379(p<0.05)$, the correlation coefficient of SRSF8 is $0.437(p<0.02)$, and the correlation coefficient of SRSF11 is $0.375(p<0.05)$ (Fig. 4C). Therefore, we preliminarily inferred that SRSF1, SRSF11 may be the upstream regulator of SRA1. Also, western-blot results indicate that the expression of SRSF1 in HCCLM3 cells was significantly higher than that in normal LO2 cells and low-transformed hepatoma cells, HepG2 and Huh-7 (Fig. S1B). Then, we overexpressed and knocked down SRSF1, and SRSF11 in HCCLM3 cells. We employed RT-qPCR to detect the overexpression and knockdown efficiency of SRSF1, and SRSF11 (Fig. S2A, B and Fig. S3A, B). We found that knockdown of SRSF1 significantly increased the expression of SRA1-S while the over-expression SRSF1 significantly increased the expression of SRA1-L in HCCLM3 cells. SRSF8 and SRSF11 had no significant effect on the alternative splicing of SRA1 in HCCLM3 cells (Fig. 4D).

Also, we measured the expression of the long isoforms of SRA1 in cells stably transduced with SRSF1 and deletion mutants RRM1, RRM2 and RS by RT-PCR. We found that the overexpression of SRSF1 can promote the inclusion of exon 3 of SRA1 compared with the empty vector control. $\triangle R S$ and $\triangle R R M 2$ mutants promoted a similar increase in the inclusion of exon 3 , while $\triangle R R M 1$ mutants didn't increase the inclusion of exon 3 of SRA1 (Fig. S4A). These results suggest that SRSF1 can regulate the alternative splicing of SRA1 and promote the production of SRA1-L.

\section{SRSF1 enhances splicing of SRA1 exon 3 through its interaction with "GGAACAGGCAUUGGAAGA" sequence in exon 3}

To further illustrate that SRSF1 is involved in the alternative splicing of SRA1 pre-mRNA, we constructed an SRA1-minigene vector. We transfected SRA1-minigene into HCCLM3 cells overexpressing SRSF1 and knocking down SRSF1. Then we detected the expression of exogenous SRA1-L and SRA1-S by RT-PCR. The results of RT-qPCR (Fig. 4E) showed that $S R A 1-S$ in minigene was significantly increased in HCC cells where SRSF1 was knocked down compared with non-treated cells. Consistent with expectations, we observed the opposite result in HCC cells by overexpressing SRSF1. Overexpression of SRSF1 promoted the generation of SRA1-L. 


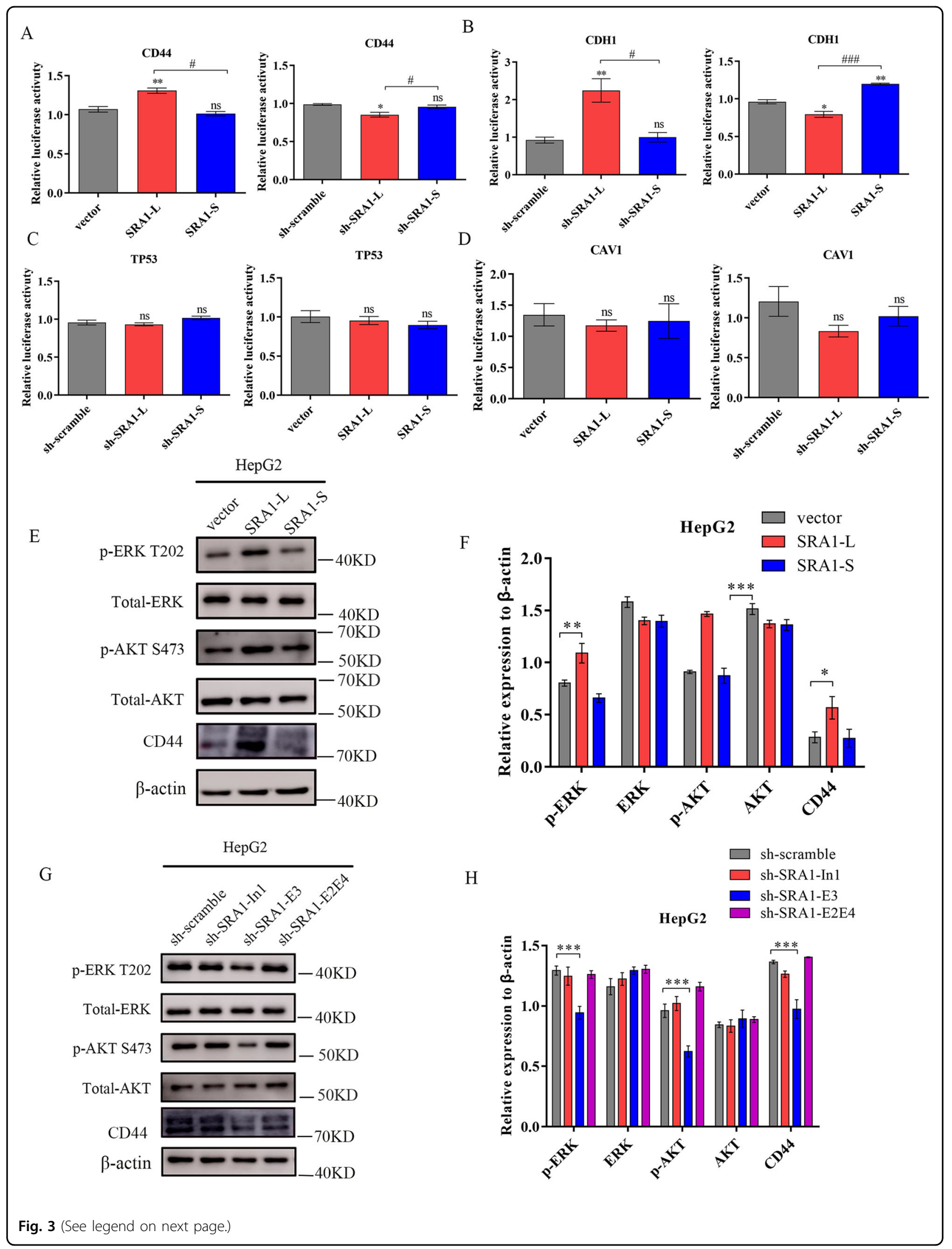


(see figure on previous page)

Fig. 3 SRA 1 isoforms have different regulatory effects on gene transcription. A The effect of SRA1 on CD44 Transcription. B The effect of SRA1 on CDH1 Transcription. C The effect of SRA1 on TP53 Transcription. D The effect of SRA1 on CAV1 Transcription. E, $\mathbf{F}$ The effect of overexpressed SRA1-L/S on downstream CD44, AKT, and ERK signaling pathway proteins was detected in HepG2 cells by western-blot. G, H The effect of knocked down SRA1-E3, SRA1-E2E4, and SRA1-In1 on downstream CD44, AKT, and ERK signaling pathway proteins was detected in HepG2 cells by western-

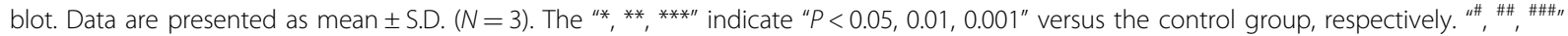
represents " $P<0.05,0.01,0.001 "$.

SRSF1 is a typical splicing factor that preferentially binds to exonic sequences to promote exon inclusion ${ }^{27}$. Then we found that GAAGA $^{28,29}$ and GGAGGA ${ }^{27,30}$ sequence was present in the exon3 of SRA1. So, we designed primers in exon 2, exon 3, and exon 4, and performed CLIP to detect whether SRSF1 binds to exon 3. The result of RT-PCR indicated that the binding of SRSF1 to exon 3 was stronger than others (Fig. 4F). At the same time, we verified whether SRSF1 and SRA1 were bound by MS2-RIP experiments. The result of MS2-RIP show that SRSF1 and SRA1 pre-mRNA are bound before splicing (Fig. $4 \mathrm{H}$ ). Through the analysis of the SRA1 base sequence, we found two potential binding sequences for SRSF1: (GAGGCTGTGATGGAGGA, E3-1) and (GGAACAGGCATTGGAAGA, E3-2) in exon 3 (Fig. $5 \mathrm{G})$. To determine the interactions between the two potential binding sites in exon 3 and SRSF1, RNA-pulldown assays were performed. We synthesized biotinylated SRA1 RNA oligomers and the mutations of potential binding sites. The GAGGCUGUGAUGGAGGA (E3-1-WT) sequence in exon 3 was mutated to CUCCCUCUCUUCCUCCU (E3-1-Mu). The GGAACAGGCAUUGGAAGA (E3-2-WT) sequence in exon 3 was mutated to CCUUCUCCCUUUCCUUCU (E32-Mu). Data showed that SRSF1 could combine with E3-2WT, but not E3-1-WT, E3-1-Mu, or E3-2-Mu (Fig. 4G). At the same time, we mutated "GGAACAGGCAttGGAAGA" in SRA1-mini-MS2-WT to "ggaCcCggcCttggaaga" (SRA1mini-MS2-Mu). When the binding site of SRSF1 in the minigene was artificially mutated, SRSF1 could not bind to the pre-mRNA of SRA1(Fig. 4H). The above results indicated that SRSF1 promotes the inclusion of exon 3 by binding to the "GGAACAGGCATTGGAAGA" sequence in exon 3, thus regulating the alternative splicing of exon 3 of SRA1.

\section{SRSF1 promoting the migration of HCC cells partially depends on the SRA1-L}

The above research results indicated that SRSF1 can regulate the alternative splicing of SRA1 and promote the expression of SRA1-L. Also, SRA1-L and SRA1-S can affect the migration of HCC cells. Our results of wound healing showed that overexpressed SRSF1 can increase the migratory abilities of HepG2 and HCCLM3 cells. Meanwhile, the depletion of SRSF1 significantly inhibited this ability (Fig. 5A, B). Also, trans-well experiments showed that overexpression of SRSF1 enhanced the ability of cell migration, and the depletion of SRSF1 significantly inhibited the ability of cell migration (Fig. 5E, F). And overexpression of SRSF1 and knockout of SRA1-L reduced the invasion of hepatoma cells, compared with HepG2 cells with overexpression of SRSF1 alone (Fig. 5E). Similarly, knockdown SRSF1 and overexpress SRA1-L are more aggressive than knockdown SRSF1 alone (Fig. 5F).

Western-blot results indicate that over-expression of SRSF1 can promote the expression of p-AKT, CD44, and $\mathrm{p}$-ERK, and the depletion of SRSF1 significantly inhibits the expression of p-AKT, and p-ERK (Fig. 6A, B). In addition, we assessed the effects of SRSF1 overexpression or depletion on cell proliferation. The results of CCK8 showed that knocking down SRSF1 inhibited the proliferation of HepG2 and HCCLM3 cells. Overexpression of SRSF1 promoted the proliferation of HepG2 and HCCLM3 cells (Fig. S4B).

In vivo metastasis assays, HE staining showed that the lung metastatic nodules of nude mice were significantly reduced after knocking out SRSF1 or SRA1-L (Fig. 6C, D). Interestingly, the overexpression of SRA1-L reversed the inhibitory effect of down-regulated SRSF1 on lung metastasis of hepatocellular carcinoma cells. In addition, compared with the control group, the inhibitory effect of sh-SRSF1 + SRA1-S on lung metastasis of hepatocellular carcinoma cells was stronger in the experimental group (Fig. 6C, D). Immunohistochemistry showed that knockdown of $S R A 1-L$ or SRSF1 significantly reduced the expression of $\mathrm{N}$-cad, while knockdown of SRA1-L or SRSF1 significantly increased the expression of E-cad (Fig. 6E). Excitingly, overexpression of SRA1-L significantly reversed the effect of knockdown of SRSF1 on the expression of E-cad and N-cad. Besides, compared with the control group, the group of shSRSF1 and overexpression of SRA1-S more robustly inhibited the expression of $\mathrm{N}$-cad and promoted E-cad (Fig. 6E). Therefore. SRSF1 may affect the metastasis of HCC by regulating the splicing of SRA1.

\section{Discussion}

Abnormal alternative splicing is often detected in the occurrence and development of hepatocellular carcinoma $^{31}$. For examble, Zhang et al found that hnRNPU regulates the alternative splicing of DIS3L2 to promote the development of liver cancer ${ }^{32}$. Friedman et al found that KLF6 regulates the alternative splicing of Ras to promote the proliferation of hepatocellular carcinoma cells $^{33}$. In this study, We found that SRSF1 regulates the alternative splicing of SRA1 to promote the migration of 


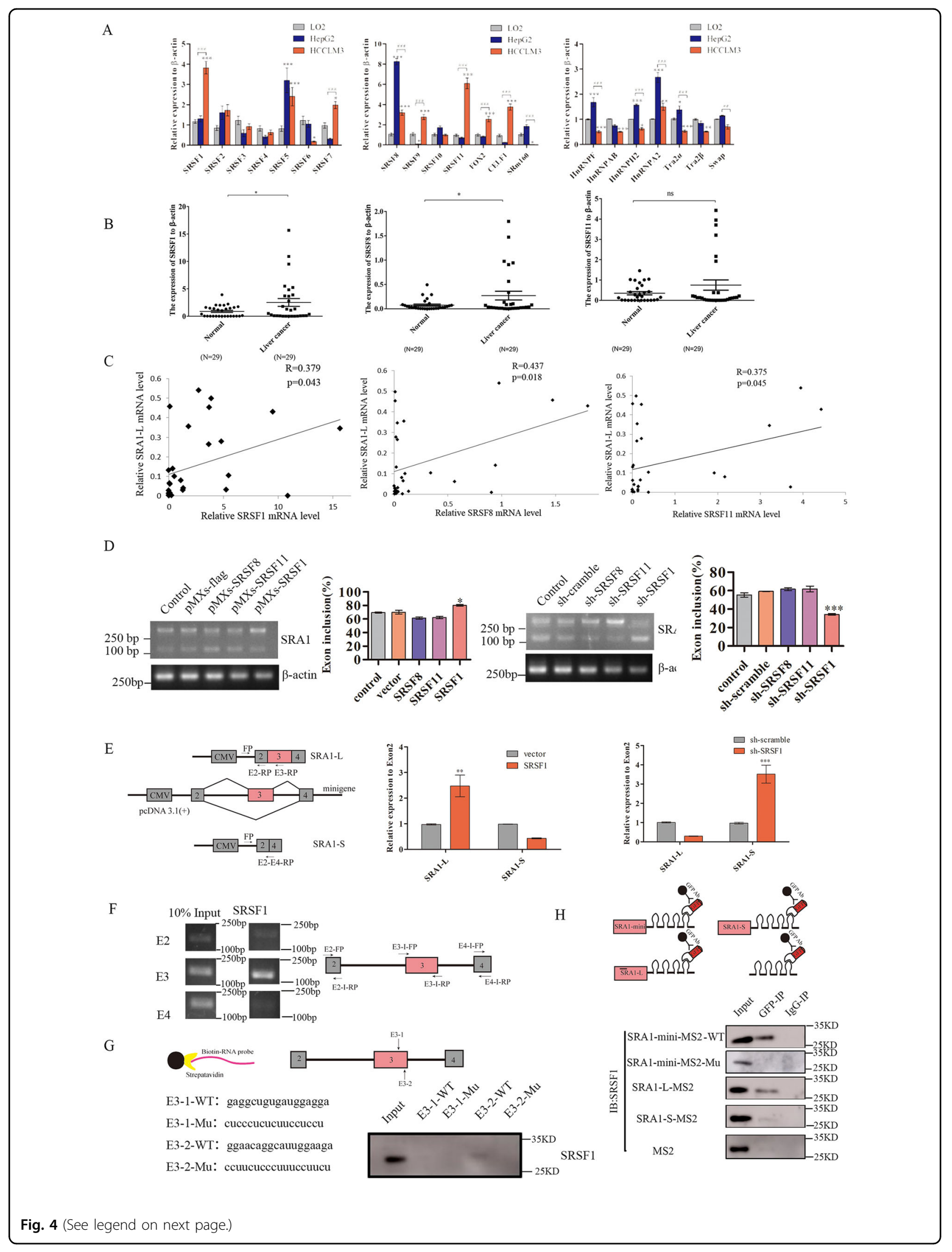


(see figure on previous page)

Fig. 4 SRSF1 regulates alternative splicing of SRA1 exon 3 by binding to "GGAACAGGCATTGGAAGA" sequence in exon 3 to promote exon inclusion. A RT-qPCR was used to detect the expression levels of different splicing factors in LO2, HepG2, and HCCLM3 cells. B Detect the mRNA expression of SRSF1, SRSF8, SRSF11 and SRA1-L in HCCS. C Correlation analysis of the expression levels of SRSF1, SRSF8, SRSF11 and SRA1-L. Use SPSS analysis tools for correlation analysis of gene expression. D Alternative splicing of exon 3 of SRA1 was examined in HCCLM3 with over-expression or knockdown of SRSF1, SRSF8, and SRSF11 by RT-PCR. E Graphical and specific primer design sites for pCDNA3.1-SRA1-minigene. The primers for the internal reference of exogenous SRA1-minigene were CMV-FP (FP), exon 2-RP (E2-RP), exon 3-RP (E3-RP) and exon 2/exon 3 splice junction-RP (E2-E4RP), and the primers for RT-qPCR detection of exogenous SRA1 isoforms in HCCLM3. $\mathbf{F}$ The primers used to detect the binding segment of SRSF1 and its positions in SRA1 pre-mRNA in the CLIP experiment. The CLIP assays were applied to detect the binding between SRSF1 and SRA1 pre-mRNA. G The RNA fragment sequences utilized in the RNA pulldown assay and its positions in the SRA1 pre-mRNA. RNA pulldown assays were used to detect the binding between SRSF1 and the biotinylated SRA1 RNA fragments. $\mathbf{H}$ Graphical representation of the MS2-GFP-IP system and its mode of operation. The effect of SRSF1 binding to SRA1 was investigated by the MS2-GFP-IP system. Data are presented as mean \pm S.D. $(N=3)$. The *****,**" indicate " $P<0.05,0.01,0.001$ " versus the control group, respectively.

hepatocellular carcinoma cells. Both SRSF1 and SRA1-L enhance cell growth, invasion, and migration in HCC cells (Fig. 7B).

The recognition of RNA binding sites by SRSF1 varies, with binding sequences including: RGAAGAAC, AGGACAGAGC, AGGACRRAGC, AACAGGACAA, AA $(\text { AGGACAA })_{2} A A$, SRSASGA $(S=G / C, R=A / G)^{30,34,35}$. In this paper, we found that SRSF1 regulates the splicing of SRA1 by binding to "GGAACAGGCAUUGGAAGA" on exon 3 of SRA1. Combined with the results of this article, we compared the sequence characteristics of SRSF1 preference binding, and found that the AG content in the sequence is higher, and the sequence AG seems to be interlaced. So, study of the pre-mRNA recognition site of SRSF1 could provide a molecular mechanism for examining the SRSF1- mediated splicing of target genes.

There are many hypotheses about the molecular mechanism of SRSF1-mediated splicing regulation. It has been reported that SR protein binds to exon ESE, directs U2AF protein to 3 'splice site and U1snRNP to $5^{\prime}$ splice site $^{36-38}$. Also, SR protein can promote splice assembly ${ }^{39}$. MS2-IP experiment shows that overexpression of SRSF1 increases the binding of U1-U6 to pre-mRNA (Fig. 7A). SRSF1 seems to play such a role in regulating SRA1 splicing, after SRSF1 binds to SRA1pre-mRNA, it recruits U1-U6 to bind to Pre-mRNA, increases the use of proximal 3'and 5', and promotes the inclusion of SRA1 exon 3. Alternative splicing of pre-mRNA is a complex process, and many cofactors are involved. In the splicing process of SRA1, SRSF1 may play a major role, and it also needs other cofactors to complete the splicing of SRA1 together. So, beyond the involvement of SRSF1, other splicing factors may regulate the splicing of SRA1.

In addition, our data show that the binding ability of SRA1-L with PPAR- $\gamma$ is stronger than that of SRA1-S (Fig. S4C, D). The PPAR- $\gamma$ binding element (PPRE) exists in the $C D 36$ promoter region and enadbles PPAR- $\gamma$ to transcriptionally regulate $C D 36^{40}$. The article reported that the core sequence of PREE in the CD36 promoter is AAGTCAG-AGGTCA. Yet, the core sequence of PREE is not fixed, unlike GGGGGA-A-AGGTTA in the Cidea promoter and AGGGCA-C-AGGAGA in Fatp ${ }^{41}$. Through sequence comparison, we found that there are similar sequences in CD44, for example: AGGGCA-G-AGCTGG, AGGGCA-ACATCAG, and GGGGGA-C-TGGAGT. We speculate that $S R A 1-L$ can regulate the transcription of $C D 44$ by binding to PPAR- $\gamma$. This will be the focus of our follow-up research.

In summary, our study identified an important oncogene, SRSF1, and an abnormal alternative splicing event, inclusion or skipping of SRA1 exon 3. Both isoforms of SRA1 play vital roles in cell invasion and migration and serve as prognostic biomarkers and therapeutic targets for liver cancer.

\section{Materials and methods}

\section{Clinical specimens and cell cultures}

Clinical research was performed according to written approval obtained from the first affiliated hospital of Jinan University (Guangzhou, China). HCCs and their matched adjacent noncancerous tissues were provided by the first affiliated hospital of Jinan University (Guangzhou,China). All clinical specimens were collected with the informed consent of the patients. Human embryonic kidney cell (293 T), human liver cell (LO2), and HCC cell lines (HepG2, Huh-7) were provided and identified by Guangzhou Institute of Biomedicine and Health. Human high metastatic HCC cells cells (HCCLM3) were purchased from Keygene Biotechnology Company Limited. HepG2, Huh-7, and HCCLM3 cells were cultured in RPMI-1640 (Gibco, Carlsbad, CA, USA), 293 T cells were maintained in DMEM (Gibco, Carlsbad, CA, USA). All media were supplemented with $10 \%$ FBS (Gibco, Carlsbad, CA, USA) and 1\% penicillin/streptomycin (Beyotime, China). All cells were cultured at $37^{\circ} \mathrm{C}$ in an atmosphere containing $5 \% \mathrm{CO}_{2}$.

\section{Bioinformatics analysis}

Bioinformatics web Rapmap ${ }^{42}$, RBpdb ${ }^{43}$, ESEfinder (c) ${ }^{44,45}$ were used to predict potential SRSF1 binding sites on $S R A 1$ pre-mRNA. In addition, we predicted the coding 


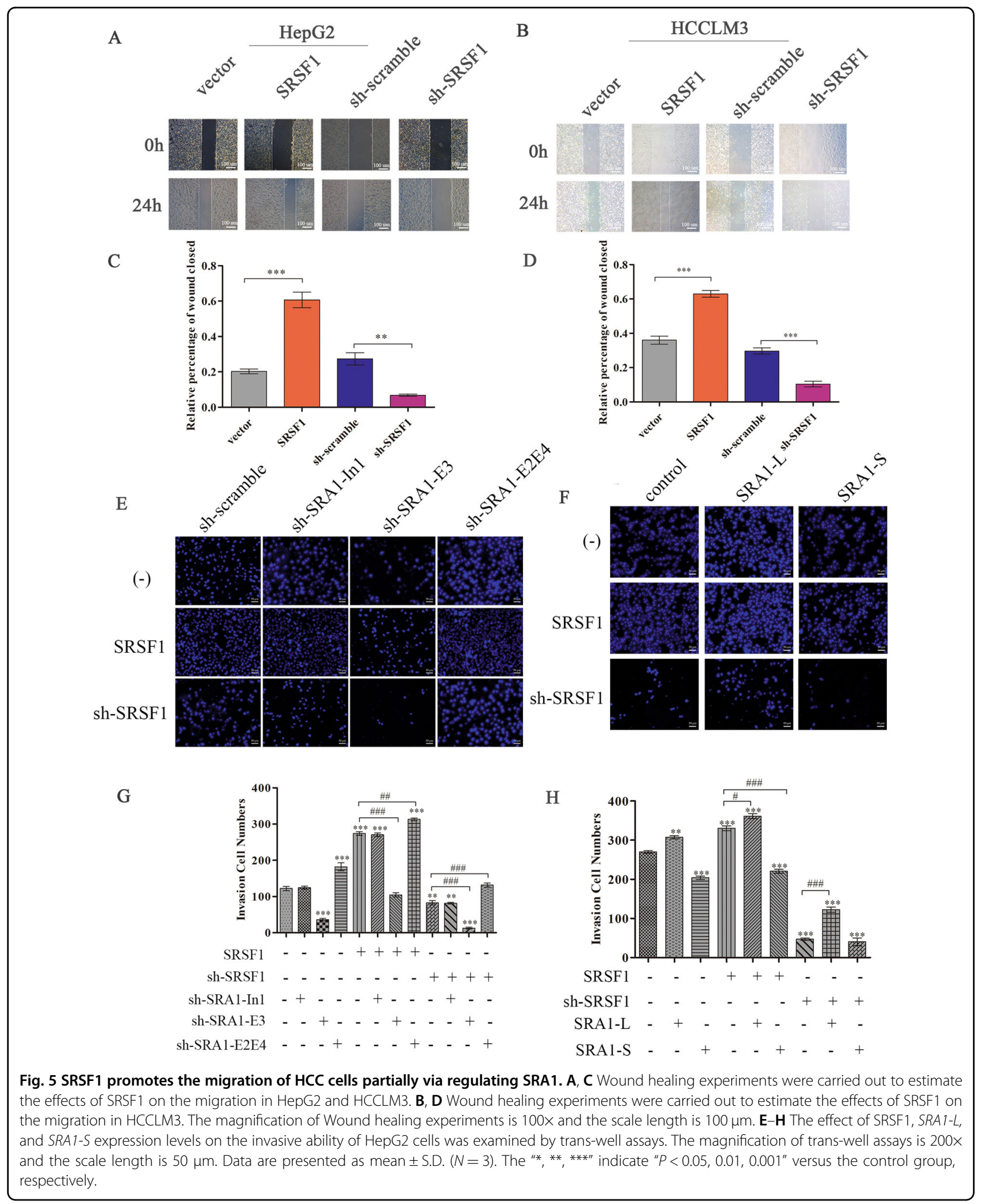

potential of SRA1-S by CPAT ${ }^{46}$. The UALCAN database ${ }^{24}$ was employed to predict the correlation between the expression of different genes and liver cancer stages.

\section{Plasmid construct}

The coding sequences (CDS) of SRSF1 (GenBank accession no. NM_006924), SRSF8 (GenBank accession 


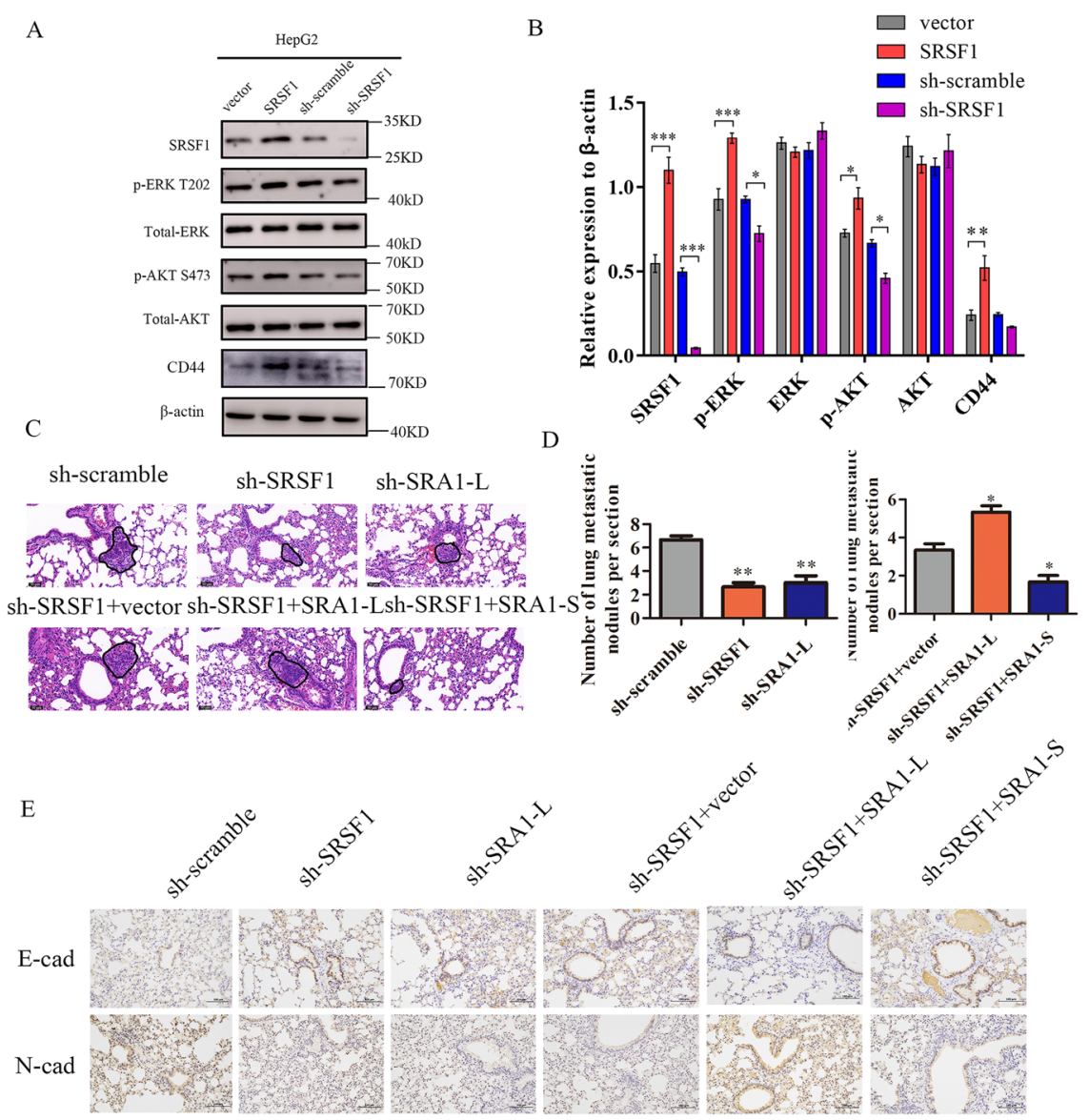

Fig. 6 In vivo, SRSF1 promotes the metastasis of HCC cells. A, B The effect of SRA1 on downstream CD44, AKT, and ERK signaling pathway proteins was detected in HepG2 cells by western blot. C, D HE staining was used to analyze the number of pulmonary metastases in different groups of nude mice. The magnification of HE is $200 \times$ and the scale length is $50 \mu \mathrm{m}$. $\mathbf{E}$ The expression level of N-cad and E-cad in tumor tissues was analyzed by immunohistochemistry. The magnification of immunohistochemistry is $100 \times$ and the scale length is $100 \mu \mathrm{m}$. Data are presented as mean \pm S.D. $(N=3)$. The ${ }^{\prime *},{ }^{* *},{ }^{* * * \prime}$ indicate " $P<0.05,0.01,0.001 "$ versus the control group, respectively.

no. NM_032102) and SRSF11 (GenBank accession nos. NM_001350605), and the full-length of SRA1-S and SRA1-L (GenBank accession no. NM_001035235) were PCR-amplified and subcloned into the pMD19-T Vector (TaKaRa, Japan), then subcloned into the Pme I site of the pMXs-Flag vector (Thermo Scientific, USA) named pMXs-SRSF1, pMXs-SRSF8, pMXs-SRSF11, pMXsSRA1-L, pMXs-SRA1-S respectively. MS2-12× fragment was PCR-amplified by PrimeSTAR® HS from pSLMS2-12× (Addgene, USA) using primers pcDNA3.1MS2-HR-FP and pcDNA3.1-MS2-HR-RP. Next, homologous recombination kits were exploited to clone the fragment into EcoR V and Xho I site of pcDNA3.1 (+), named pcDNA3.1-MS2. The full-length of SRA1-S, SRA1$L$ was PCR-amplified from pMXs-SRA1-L, pMXs-SRA1-S using primers pcDNA3.1-SRA1-MS2-HR-FP and pcDNA3.1-SRA1-MS2-HR-RP, and cloned into Xho I site of the pcDNA3.1-MS2 vector using homologous recombination kits, named SRA1-L-MS2, SRA1-S-MS2. The
Minigenes were constructed by amplifying genomic sequences spanning exons 2 to 4 of the SRA1 gene using primers SRA1-mini-HR-FP and SRA1-mini-HR-RP and, subsequently, using homologous recombination kits cloned the fragment into EcoR $V$ and Xho I site of the pcDNA3.1 (+) vector, named SRA1-mini. MS2-12× fragment was PCR-amplified pSL-MS2-12× (Addgene, USA) using primers SRA1-mini-MS2-HR-FP/RP, subsequently, using homologous recombination kits cloned the fragment into Xho I site of SRA1-mini.

Short hairpin RNA (shRNA) sequences were designed to suppress the expression of SRSF1, SRSF8, SRSF11, SRA1E3, SRA1-E2E4, and SRA1-In1. A scrambled shRNA was used as a negative control. After annealing, insert a doublestranded oligonucleotide between the Hind III and Bgl II restriction sites of the pSuper-Retro vector. The upstream and downstream primers were specifically designed to delete the RRMs and RS fragment of SRSF1. PCR amplification was carried out using pMXs-SRSF1 plasmid as a 

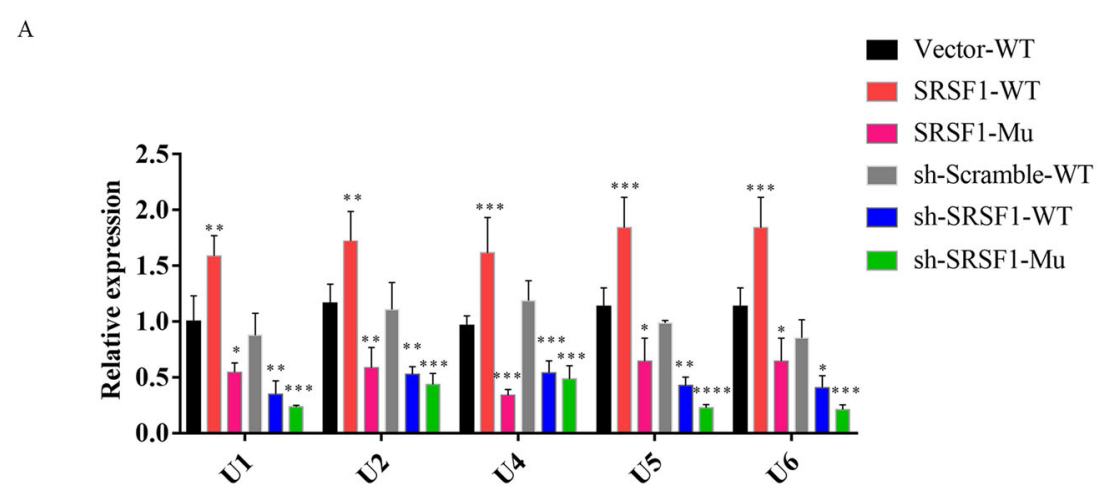

B

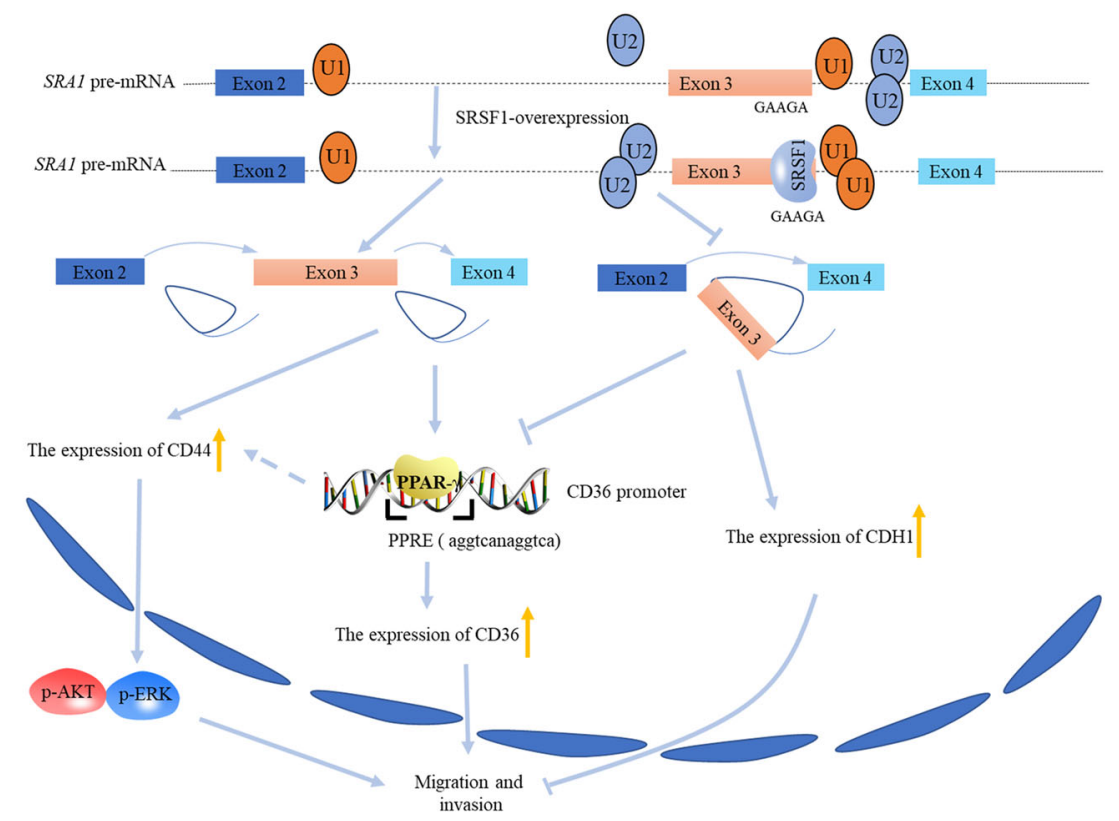

Fig. 7 SRSF1 regulates the alternative splicing of SRA1 and promotes the metastasis of liver cancer. A The effect of SRSF1 on the ability of snRNAs to bind to SRA1 pre-mRNA was studied by the MS2-GFP-RIP system. B The effect of the SRSF1-SRA1 signal axis on the migration and invasion of the HCC cells.

template. After adding solution I (TaKaRa, Japan) overnight connection, $1 \mu \mathrm{l} D p n$ I was added to $10 \mu \mathrm{l}$ of the connection product, and after $1 \mathrm{~h}$ at $37^{\circ} \mathrm{C}, 10 \mu \mathrm{l}$ of the reaction product was 4 transformed into $\mathrm{DH} 5 \alpha$ cells.

The primers employed in this paper were designed by our group with reference to the NCBI sequences and were synthesized by Guangzhou Qsingke Biological Company. The primers are shown in Supplementary Table. 1.

\section{Transfection and Retrovirus infection}

All the plasmids were transfected into cells using Lipofectamine 6000 reagent (Beyotime, China) according to the manufacturer's protocol. Retrovirus viral particles were produced in $293 \mathrm{~T}$ cells transfected with the pCL retroviral packaging plasmid in DMED media. Infectious lentivirus was harvested at $48 \mathrm{~h}$ post-transfection and filtered through $0.45 \mu \mathrm{m}$ nitrocellulose filter. The viral supernatant was employed for infecting HepG2 or HCCLM3 cells after supplementation with $8 \times 10^{-6} \mathrm{mg} /$ $\mathrm{ml}$ Polybrene (Sigma-Aldrich, USA). The cells infected with viruses generated from transfection with pSUPERScramble (Scramble) or pMXs-Vector were used as control groups. For cells infected with shRNA retrovirus, cells with stable knockdown of the target genes could be obtained after 10 days of selection with $2 \times 10^{-3} \mathrm{mg} / \mathrm{ml}$ Puromycin (Thermo Scientific, USA).

\section{Semi-quantitative RT-PCR and real-time qPCR (RT-qPCR) analysis}

The Trizol (Beyotime, China) was applied to extract total RNA. Cytoplasmic and nuclear RNA was isolated and purified using the Nuclear and Cytoplasmic Protein 
Extraction Kit (Beyotime, China) according to the manufacturer's instructions. Semi-quantitative RT-PCR was performed with Golden Star T6 Super PCR Mix (TsingKe, China), The PCR products were separated by $2 \%$ agarose gel electrophoresis. The ImageJ software was employed to quantify. Exon inclusion (\%) = Gray value of long isomer / (gray value of long isomer + gray value of short isomer). Real-time qPCR was performed in the LightCyler 480 System (Roche, Switzerland) using SYBR ${ }^{\circledR}$ Premix Ex $\mathrm{Taq}^{\mathrm{TM}}$ (TaKaRa, Japan) and the gene-specific primers. $\beta$-actin was employed as an endogenous control. The relative expression of RNA was calculated using the $2^{-\Delta \Delta \mathrm{Ct}}$ method.

\section{Cell proliferation assays}

For cell proliferation assays, 3000 cells were seeded into 96-well plates. Cell proliferation was assessed using the CCK8 (Beyotime, China) according to the manufacturer's protocol. Then $\mathrm{OD}_{450}$ values of each well were measured by Synergy TM HT (BioTeK, USA).

\section{Wound-healing assays and invasion assays}

For wound-healing assays, linear scratch wounds (in triplicate) were created on the confluent cell monolayers using a $200 \mu \mathrm{l}$ pipette tip. To remove cells from the cell cycle prior to wounding, cells were maintained in the serum-free medium. Scratch healing rate $=\left(\mathrm{W}_{0 \mathrm{~h}}-\mathrm{W}_{24 \mathrm{~h}}\right) / \mathrm{W}_{0 \mathrm{~h}}$. The transwell insert (Corning, USA) was ultized as per the manufacturer guideline. $3 \times 10^{5}$ cells were added to the upper wells separated by an $8 \mu \mathrm{m}$ pore size PET membrane with a thin layer of Matrigel basement membrane matrix for invasion. After being incubated $24 \mathrm{~h}$, the non-migrated cells were removed from the top of the membrane with Cotton Cswab, and then the membranes were stained with DAPI (Beyotime, China) for $5 \mathrm{~min}$ after removing. Cells were visualized under a fluorescent microscope (ZEISS, AXIOVERT A1). Six random fields were captured at $200 \times$ magnification.

\section{Western blot analysis}

The cells were lysed with RIPA buffer, and then the protein concentration was detected by BCA protein detection kit (Beyotime, China). $20 \mu \mathrm{g}$ of whole-cell lysates was separated by SDS-PAGE and then transferred to a preactivated Polyvinylidene Fluoride membrane (PVDF), the membrane was blocked for $1 \mathrm{~h}$ in TBST buffer (TBS containing $0.1 \%$ Tween 20 ) containing $5 \%$ non-fat dry milk followed by an overnight incubation with primary antibody diluted. AKT primary antibody (C67E7) and phosphorylated Ser473 AKT primary antibody $(4060 \mathrm{~T})$ were purchased from Cell Signaling Technology Company. ERK primary antibody (AF1051), phosphorylated Thr202 ERK primary antibody (AF1891) and $\beta$-actin primary antibody (AF0003) were purchased from Beyotime Company. SRSF1 primary antibody (NHA3445) was purchased from
Novogene Company. After extensive washing, the blot was incubated with a secondary antibody overnight. Finally, the membranes were washed thrice with TBST and visualized by BeyoECL Moon kit (Beyotime, China). Imaging was then performed using a biochemi-luminometer (General Electric, AI600) and Image J software was ultized to quantify the bands' grayscale.

\section{RNA pull-down assays}

The RNA pull-down assays were carried out by synthesized biotinylated SRA1 RNA (Tsingke, China) as a probe. The Pierce ${ }^{\mathrm{TM}}$ Magnetic RNA-Protein Pull-Down Kit (Thermo Scientific, USA) was used to perform the experiment. For each sample, $50 \mu \mathrm{l}$ streptavidin magnetic beads were engrafted to capture the biotin-labeled RNA. The products of RNA and nuclear protein complexes were washed and analyzed by western blot.

\section{RNA immunoprecipitation assays}

pcDNA3.1-MS2-SRA1-mini, pcDNA3.1-MS2-SRA1-L, pcDNA3.1-MS2-SRA1-S and pcDNA3.1-MS2 were cotransfected with pMS2-GFP (Addgene, USA) into HepG2 cells. Cell lysates were utilized to perform RIP experiments using a GFP antibody (Beyotime, China) coupled with protein $\mathrm{G}$ magnetic beads ( $50 \mu \mathrm{l}$ per reaction) $48 \mathrm{~h}$ later. Subsequently, $500 \mathrm{ul}$ of RIPA was added to the magnetic bead complex. Finally, the protein was analyzed by western blot.

\section{Cross-link immunoprecipitation (CLIP)}

CLIP was performed to certify the binding between SRSF1 and SRA1. Briefly, RNA and protein complexes were cross-linked with $1 \%$ formaldehyde in ultraviolet cross-linking was performed at $400 \mathrm{~mJ} \mathrm{~cm}^{-2}$ for $10 \mathrm{~min}$. Immunoprecipitation was done with $10 \mu \mathrm{g}$ anti-SRSF1 antibody (Novogene, China) or either nonspecific IgG coupled with $50 \mu \mathrm{l}$ protein $\mathrm{G}$ magnetic beads. Then, extracting RNA from complexes and synthesizing cDNA with random primers. Finally, RNA enrichment was measured by RT-PCR with primers specific for SRA1 mRNA.

\section{Luciferase reporter assays}

pGL3-CDH1, pGL3-CD44, pGL3-TP53, pGL3-CAV1, and phRL-SV40 were co-transfected into overexpressed or knocked down SRA1 isoform HepG2 cells by using Lipofectamine 6000 reagent (Beyotime, China) according to the manufacturer's protocol. $48 \mathrm{~h}$ after transfection, the cells were lysed, and luciferase activity was measured using a Dual-Luciferase Reporter Gene Assay Kit (Beyotime, China).

\section{In vivo metastasis assays}

3-week-old male nude mice (BALB/c-nu/nu) $(N=48)$ were purchased from Beijing HFK Biotechnology Co, Ltd. The animals were maintained under Specific Pathogen Free 
(SPF) conditions and were provided food and water. The animals were randomly divided into 6 groups with 8 animals in each group. Six different groups (sh-scramble, shSRSF1, sh-SRA1-L, sh-SRSF1 + pMXs-flag, sh-SRSF1 + pMXs-SRA1-L, sh-SRSF1 + pMXs-SRA1-S) of HCCLM3 cells $\left(1 \times 10^{6}\right.$ cells in $100 \mu$ l serum-free media) were injected into the tail vein of 4-week-old BALB/C female nude mice (weighing $18-22 \mathrm{~g}, N=48$ ). After 30 days of normal feeding, lungs were harvested at necropsy and fixed in paraformaldehyde. All animal procedures were performed in accordance with the Jinan University Experimental Animal Care Commission. All animal experiments comply with ARRIVE guidelines, and are carried out in accordance with the U.K. Animals (Scientific Procedures) Act, 1986, EU Directive 2010/63/EU for animal experiments, and the National Institutes of Health guide for the care and use of Laboratory animals (NIH Publications No. 8023, revised 1978). No blinding was used in animal experiments.

\section{HE (Haematoxylin-Eosin)}

The lungs were fixed in $4 \%$ paraformaldehyde and transferred to $70 \%$ ethanol, then embedded in paraffin. Before immunostaining, 4- $\mu \mathrm{m}$-thick lung tissue sections were dewaxed in xylene, rehydrated through decreasing concentrations of ethanol, and washed in PBS. Then stained with hematoxylin and eosin (H\&E). After staining, sections were dehydrated through increasing concentrations of ethanol and xylene.

\section{Immunohistochemistry (IHC)}

For immunohistochemical analysis of E-Cadherin, NCadherin tissue sections were prepared, as described previously ${ }^{47}$. The primary antibody used was E-Cadherin Mouse Monoclonal Antibody (1:100) (Beyotime, AF0138) and N-Cadherin Rabbit Polyclonal Antibody (1:200) (Beyotime, AF0138).

\section{Statistics analysis}

Using SPSS Statistical Package version 24 (SPSS Inc, Chicago, Illinois) to analyze the correlation of data. Data from the experiments with one experimental group were presented as means \pm Standard Deviation (SD) (all experiments were performed in triplicate or more). One-way (or two-way) analysis of variance (ANOVA) and Tukey's posthoc analysis was exploited to evaluate statistical significance. ${ }^{*} P<0.05,{ }^{* *} P<0.01$ or ${ }^{* * * *} P<0.001$ was considered to indicate statistical significance, "\#" represents $P<0.05$, "\#\#" represents $P<0.01$, and “\#\#” represents $P<0.001$, ns means no significance. When representative figures are shown, these are representative of three independent repeats.

\section{Acknowledgements}

Thanks to research group of Zhimin Zhang (School of Pharmacy, Jinan University) for providing DNA polymerase (Takara), and other biological reagents.

\section{Author details}

${ }^{1}$ Institute of Genomic Medicine, College of Pharmacy, Jinan University, Guangzhou, China. ${ }^{2}$ International Cooperative Laboratory of Traditional Chinese Medicine Modernization and Innovative Drug Development of Chinese Ministry of Education (MOE), College of Pharmacy, Jinan University, Guangzhou, China. ${ }^{3}$ University of Chinese Academy of Science, Beijing, China. ${ }^{4}$ Department of Internal Medicine, Morsani College of Medicine, University of South Florida, Tampa, FL, USA. ${ }^{5}$ Brown School of Social Work, Washington University in St. Louis, St. Louis, MO, USA. ${ }^{6}$ College of Life Sciences, Zhengzhou University, Zhengzhou, Henan, China. ${ }^{7}$ Department of General Surgery, The First Affiliated Hospital, Jinan University, Guangzhou, China

\section{Author contributions}

Conceptualization: F.W., Q.Li. Supervision: F.W., S.L., B.Z. Reagent Generation: F. W., L.H. Experiments: S.L., B.Z., Z.Z., L.S., H.L., H.L., Y.C. Analysis: S.L., B.Z., A.C., M.B., Q.Z. and H.S. Manuscript writing: S.L., B.Z. Collect clinical samples: K.L., Q.Li.

\section{Funding}

This work was supported by the Traditional Chinese Medicine Bureau of Guangdong Province (NO. 20141065); Science and Technology Innovation Project from the Department of Education of Guangdong Province (NO. 2012KJCX0017); Natural Science Foundation of Guangdong Province (NO. 2017A030313561 and 2018A0303130253); High-level University Construction Project; Guangzhou Science and Technology Program (NO. 202002030087); Administration of Traditional Chinese Medicine of Guangdong Province (NO. 20201078) and the National Natural Science Foundation of China (NO. 82074064).

\section{Ethics statement}

The animal study was approved by Laboratory Animal Ethics Committee Jinan University. All animal experiments was performed in accordance with the Declaration of Helsinki.

\section{Conflict of interest}

The authors declare no competing interests.

\section{Publisher's note}

Springer Nature remains neutral with regard to jurisdictional claims in published maps and institutional affiliations.

Supplementary information The online version contains supplementary material available at https://doi.org/10.1038/s41420-021-00498-w.

Received: 2 March 2021 Revised: 4 April 2021 Accepted: 24 April 2021

Published online: 19 May 2021

\section{References}

1. Bray F. et al. Global cancer statistics 2018: GLOBOCAN estimates of incidence and mortality worldwide for 36 cancers in 185 countries. CA Cancer J. Clin. https:/doi.org/10.3322/caac.21492 (2018).

2. Gupta, G. P. \& Massagué, J. Cancer metastasis: building a framework. Cell 127, 679-695 (2006).

3. Nilsen T. W., Graveley B. R. Expansion of the eukaryotic proteome by alternative splicing. Nature https:/doi.org/10.1038/nature08909 (2010).

4. Bielli, P., Bordi, M., Di Biasio, V. \& Sette, C. Regulation of BCL-X splicing reveals a role for the polypyrimidine tract binding protein (PTBP1/hnRNP I) in alternative $5^{\prime}$ splice site selection. Nucleic Acids Res. 42, 12070-12081 (2014).

5. Wan L. et al. SRSF6-regulated alternative splicing that promotes tumour progression offers a therapy target for colorectal cancer. Gut. https://doi.org/ 10.1136/gutjpl-2017-314983 (2019).

6. Shen L. et al. Skipping of exon 10 in Axl pre-mRNA regulated by PTBP1 mediates invasion and metastasis process of liver cancer cells. Theranostics. https://doi.org/10.7150/thno.42010 (2020).

7. Grelet S. et al. A regulated PNUTS mRNA to IncRNA splice switch mediates EMT and tumour progression. Nat. Cell Biol. https://doi.org/10.1038/ncb3595 (2017). 
8. Lanz R. B. et al. A steroid receptor coactivator, SRA, functions as an RNA and is present in an SRC-1 complex. Cell. https://doi.org/10.1016/S0092-8674(00) 80711-4 (1999).

9. Lanz R. B., Razani B., Goldberg A. D., O'Malley B. W. Distinct RNA motifs are important for coactivation of steroid hormone receptors by steroid receptor RNA activator (SRA). Proc. Natl Acad. Sci. USA . https://doi.org/10.1073/ pnas.192571399 (2002).

10. Hube F. et al. Alternative splicing of the first intron of the steroid receptor RNA activator (SRA) participates in the generation of coding and noncoding RNA isoforms in breast cancer cell lines. DNA Cell Biol . https://doi.org/10.1089/ dna.2006.25.418 (2006).

11. Leygue E., Dotzlaw H., Watson P. H., Murphy L. C. Expression of the steroid receptor RNA activator in human breast tumors. Cancer Res. (1999).

12. Hussein-Fikret S., Fuller P. J. Expression of nuclear receptor coregulators in ovarian stromal and epithelial tumours. Mol. Cell Endocrinol. https://oi.org/ 10.1016/j.mce.2004.08.005 (2005).

13. Lanz R. B. et al. Steroid receptor RNA activator stimulates proliferation as well as apoptosis In Vivo. Mol. Cell Biol. https://doi.org/10.1128/mcb.23.20.71637176.2003 (2003).

14. Howard J. M., Sanford J. R. The RNAissance family: SR proteins as multifaceted regulators of gene expression. Wiley Interdiscip. Rev. RNA. https://doi.org/ 10.1002/wrna.1260 (2015).

15. Karni R. et al. The gene encoding the splicing factor SF2/ASF is a protooncogene. Nat. Struct. Mol. Biol. https://doi.org/10.1038/nsmb1209 (2007).

16. Sheng J. et al. SRSF1 modulates PTPMT1 alternative splicing to regulate lung cancer cell radioresistance. EBioMedicine. https://doi.org/10.1016/j. ebiom.2018.11.007 (2018).

17. Wang J. et al. SRSF1-dependent alternative splicing attenuates BIN1 expression in non-small cell lung cancer. J. Cell Biochem. https://doi.org/ 10.1002/jcb.29366 (2020).

18. Motomura, W., Okumura, T., Takahashi, N., Obara, T. \& Kohgo, Y. Activation of peroxisome proliferator-activated receptor $\gamma$ by troglitazone inhibits cell growth through the increase of p27(Kip1) in human pancreatic carcinoma cells. Cancer Res. 60, 5558-5564 (2000).

19. Han S., Sidell N., Fisher P. B., Roman J. Up-regulation of p21 gene expression by peroxisome proliferator-activated receptor $\gamma$ in human lung carcinoma cells. Clin. Cancer Res. https://doi.org/10.1158/1078-0432.CCR-03-0985 (2004).

20. Yang F. et al. Peroxisome proliferator-activated receptor gamma ligands induce cell cycle arrest and apoptosis in human renal carcinoma cell lines. PubMed. https://doi.org/10.1111/j.1745.7254.2005.00103.x (2005).

21. Xu B. et al. Multiple roles for the non-coding RNA SRA in regulation of adipogenesis and insulin sensitivity. PLoS ONE. https:/doi.org/10.1371/journal. pone.0014199 (2010).

22. Yan Y. et al. The steroid receptor RNA activator protein (SRAP) controls cancer cell migration/motility. FEBS Lett. https://doi.org/10.1016/j.febslet.2015.11.007 (2015).

23. Eoh K. J. et al. Long non-coding RNA, steroid receptor RNA activator (SRA), induces tumor proliferation and invasion through the NOTCH pathway in cervical cancer cell lines. Oncol Rep. https://doi.org/10.3892/or.2017.6023 (2017).

24. Chandrashekar D. S. et al. UALCAN: a portal for facilitating tumor subgroup gene expression and survival analyses. Neoplasia (United States) . https://doi. org/10.1016/j.neo.2017.05.002 (2017).

25. Anand V. et al. CD44 splice variant (CD44V3) promotes progression of urothelial carcinoma of bladder through Akt/ERK/STAT3 pathways: novel therapeutic approach. J. Cancer Res. Clin. Oncol. https://doi.org/10.1007/s00432019-03024-9 (2019).

26. Geng B. et al. Chitinase 3-like 1-CD44 interaction promotes metastasis and epithelial-to-mesenchymal transition through $\beta$-catenin/Erk/Akt signaling in gastric cancer. J. Exp. Clin. Cancer Res. https://doi.org/10.1186/s13046-018-08762 (2018).

27. Pandit S. et al. Genome-wide Analysis Reveals SR Protein Cooperation and Competition in Regulated Splicing. Mol. Cell. https://doi.org/10.1016/j. molcel.2013.03.001 (2013).

28. Chen L. et al. SRSF1 Prevents DNA Damage and Promotes Tumorigenesis through Regulation of DBF4B Pre-mRNA Splicing. Cell Rep. https://doi.org/ 10.1016/j.celrep.2017.11.091 (2017).

29. Tacke, R. \& Manley, J. L. The human splicing factors ASF/SF2 and SC35 possess distinct, functionally significant RNA binding specificities. EMBO J. 14 3540-3551 (1995).

30. Ghigna, $C$. et al. Cell motility is controlled by SF2/ASF through alternative splicing of the Ron protooncogene. Mol. Cell 20, 881-890 (2005).

31. Lee S. E., Alcedo K. P., Kim H. J., Snider N. T. Alternative Splicing in Hepatocellular Carcinoma. CMGH. https://doi.org/10.1016/j.jcmgh.2020.04.018 (2020).

32. Xing $\mathrm{S}$., et al. DIS3L2 promotes progression of hepatocellular carcinoma via hnRNP U-mediated alternative splicing. Cancer Res. https://doi.org/10.1158/ 0008-5472.CAN-19-0376 (2019).

33. Yea S., et al. Ras Promotes Growth by Alternative Splicing-Mediated Inactivation of the KLF6 Tumor Suppressor in Hepatocellular Carcinoma. Gastroenterology . https://doi.org/10.1053/j.gastro.2008.02.015 (2008).

34. Graveley B. R. Sorting out the complexity of SR protein functions. RNA. https:// doi.org/10.1017/S1355838200000960 (2000).

35. Tacke R., Manley J. L. Determinants of SR protein specificity. Curr. Opin. Cell Biol. https://doi.org/10.1016/S0955-0674(99)80050-7 (1999).

36. Chen M., Manley J. L. Mechanisms of alternative splicing regulation: Insights from molecular and genomics approaches. Nat. Rev. Mol. Cell Biol. https:/doi. org/10.1038/nrm2777 (2009).

37. Robberson B. L., Cote G. J., Berget S. M. Exon definition may facilitate splice site selection in RNAs with multiple exons. Mol. Cell Biol. https://doi.org/10.1128/ mcb.10.1.84 (1990).

38. Kohtz J. D., et al. Protein-protein interactions and $5^{\prime}$-splice-site recognition in mammalian mRNA precursors. Nature . https://doi.org/10.1038/368119a0 (1994).

39. Long J. C., Caceres J. F. The SR protein family of splicing factors: Master regulators of gene expression. Biochem. J. https://doi.org/10.1042/BJ20081501 (2009).

40. Nagy, L., Tontonoz, P., Alvarez, J. G. A., Chen, H. \& Evans, R. M. Oxidized LDL regulates macrophage gene expression through ligand activation of PPARY. Cell 93, 229-240 (1998).

41. Tzeng J. et al. An ideal PPAR response element bound to and activated by PPARa. PLOS ONE . https://doi.org/10.1371/journal.pone.0134996 (2015).

42. Paz I., Kosti I., Ares M., Cline M., Mandel-Gutfreund Y. RBPmap: A web server for mapping binding sites of RNA-binding proteins. Nucleic Acids Res. https://doi. org/10.1093/nar/gku406 (2014).

43. Cook K. B., Kazan H., Zuberi K., Morris Q., Hughes T. R. RBPDB: A database of RNA-binding specificities. Nucleic Acids Res. https://doi.org/10.1093/nar/ gkq1069 (2011).

44. Cartegni L., Wang J., Zhu Z., Zhang M. Q., Krainer A. R. ESEfinder: A web resource to identify exonic splicing enhancers. Nucleic Acids Res. https://doi. org/10.1093/nar/gkg616 (2003).

45. Smith, P. J. et al. An increased specificity score matrix for the prediction of SF2/ ASF-specific exonic splicing enhancers. Hum. Mol. Genet. 15, 2490-2508 (2006).

46. Wang L. et al. CPAT: Coding-potential assessment tool using an alignmentfree logistic regression model. Nucleic Acids Res. https://doi.org/10.1093/nar/ gkt006 (2013).

47. Shen $\mathrm{L}$. et al. Therapeutic effects of the rhSOD2-Hirudin fusion protein on bleomycin-induced pulmonary fibrosis in mice. Eur. J. Pharmacol. https://doi. org/10.1016/j.ejphar.2019.03.001 (2019). 